\title{
Capacitance and Inductance Matrices for Multistrip Structures in Multilayered Anisotropic Dielectrics
}

FRANCISCO MEDINA AND MANUEL HORNO, MEMBER, IEEE

\begin{abstract}
In this paper we present a unified variational approach to determine the capacitance and inductance matrices of generalized multistrip systems embedded in a multilayered iso/anisotropic dielectric lossless medium. The analysis is carried out in the spectral domain to take advantage of previously obtained recurrence relations which calculate the Green's function in the spectral domain. The method leads to a low-order system of linear equations, which is shown explicitly. Examples and comparison with previously published results have been included.
\end{abstract}

\section{INTRODUCTION}

T HE PHYSICAL behavior of uniform multiconductor transmission lines when they are used in the design of high-frequency electrical filters and couplers is well understood [1]. It is also well known that at the low-frequency end of the spectrum, a quasi-TEM approximation can be assumed even when inhomogeneous and/or anisotropic dielectrics are involved $[2,3]$. Under this assumption, the problem reduces to the determination of the Maxwell capacitance matrix of the system. Particular interest has been focused on the planar structures used in MIC technology. Numerous papers dealing with quasi-static propagation in two- or three-conductor strip systems can be found in the literature [4-8]. The more general problem of determining the Maxwell capacitance matrix for a generalized multistrip system has been treated for homogeneous [9] and nonhomogeneous [10] media. However, for planar structures embedded in a multilayered dielectric medium, the spectral-domain analysis seems to be especially suitable $[11,12]$. Recently an efficient recurrence algorithm to obtain the Green's function in the spectral domain associated with an anisotropic multilayered dielectric medium has been used to analyze several coplanar [13] and noncoplanar [14] structures. Similar work dealing with isotropic dielectrics has also been recently published [15]. The aim of the present paper is to apply variational analysis in the spectral domain in order to obtain the capacitance and inductance matrices that characterize a multistrip system of the type shown in Fig. 1. The method leads to a low-order system of linear equations whose coefficients and independent terms are explicitly shown in the work.

Manuscript recerved April 20, 1987; revised July 2, 1987. This work was supported by the Comision Asesora de Investıgación Científica y Técnica (Proy. 3028/83), Spain.

The authors are with the Departamento de Electricidad y Electronica, Facultad de Fisica, Universidad de Sevilla, 41012, Sevilla, Spain.

IEEE Log Number 8716592

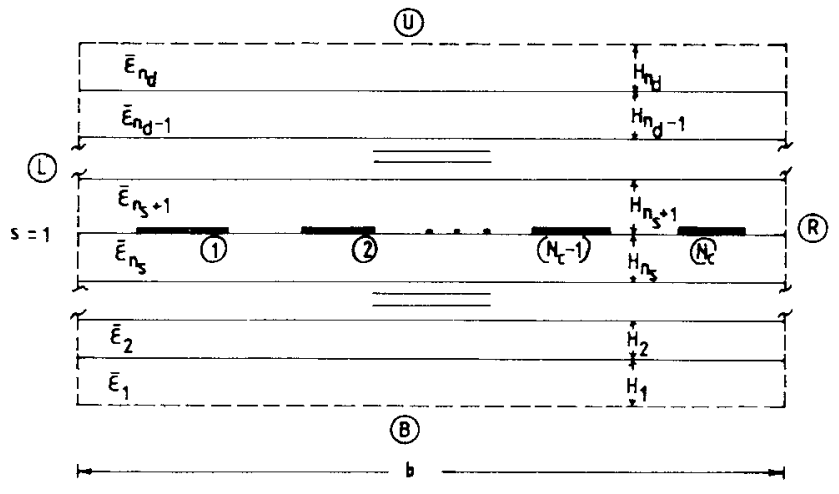

(a)

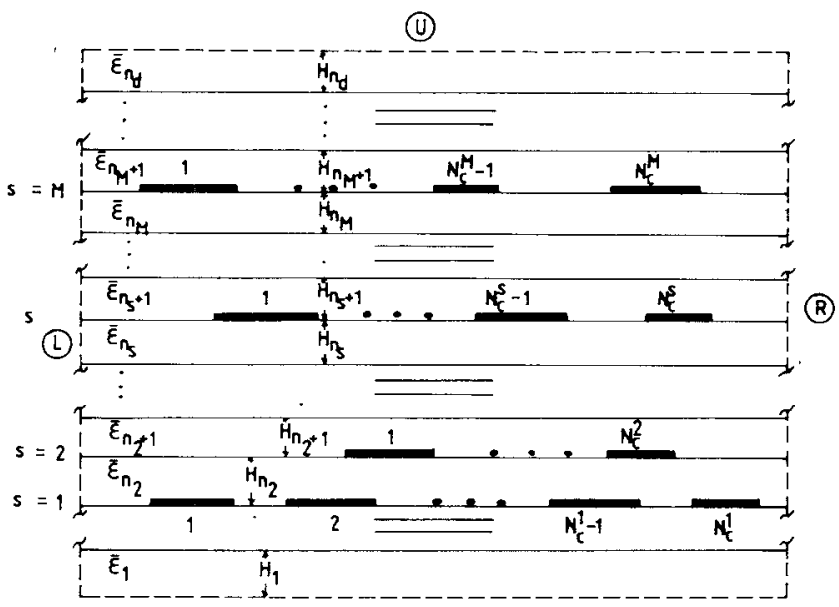

(B)

(b)

Fig. 1. (a) Cross section of generic multilayered coplanar strip lines. (b) Cross section of a general noncoplanar multistrip system. $U$ and $B$ denote electric wall, magnetic wall, or open boundary; $L$ and $R$ denote electric or magnetic wall

The choice of basis functions is discussed and several examples are introduced to show the method's strength.

\section{Statement of the Problem: SPECTRAL ANALYSIS}

Consider a system of strip lines in a stratified lossless dielectric region enclosed in a set of rectangular boundary conditions. Conductors can be coplanar (Fig. 1(a)) or 
non-coplanar (Fig. 1(b)) strips. The top and bottom shields can be considered electric walls, magnetic walls, or open boundaries. So, open geometries can be simulated by choosing an open boundary in the top shield and taking " $b$ " in Fig. 1 large enough. The permittivity of the $j$ th dielectric layer is a diagonal tensor $\overline{\bar{\varepsilon}}_{J}$. (It is important to take into account the dielectric anisotropy from a practical and theoretical point of view [16].) If nonmagnetic materials $\left(\mu_{j}=\mu_{0}\right)$ are assumed, the problem will be reduced to the determination of the capacitance matrix for the structures under consideration with $\left(\left[C_{l j}\right]\right)$ and without $\left(\left[C_{t j}^{v}\right]\right)$ substrates [3]. These two-dimensional electrostatic problems can be readily formulated in the spectral domain instead of working in the space domain [13]-[15]. The first step in the solution process is to determine Green's function in the spectral domain associated with the structure. When several dielectric layers are involved, this task may become very tedious. Nevertheless, very simple recurrence expressions to determine Green's scalar function for coplanar structures [13] (Fig. 1(a)) and Green's function matrix for noncoplanar ones [14] (Fig. 1(b)) have been published. In this way, the Fourier transforms of the potential function $\tilde{V}_{s}$ and the surface charge $\tilde{\rho}_{s}$ on the interfaces where the strips are located are related via

$$
\begin{aligned}
\tilde{V}_{s}(n) & =\tilde{G}_{s}(n) \cdot \tilde{\rho}_{s}(n) & & \text { (coplanar strips) } \\
{\left[\tilde{V}_{s}(n)\right] } & =\left[\tilde{G}_{s r}(n)\right]\left[\tilde{\rho}_{r}(n)\right] & & \text { (noncoplanar strips). }
\end{aligned}
$$

These relations will be used in the following paragraphs to derive the inductance and capacitance matrices.

\section{VARIATIONAL ANALYSIS}

In a multiconductor system such as the one described in the previous section, charges and potentials on the strips are related by means of the matrices $[C]$ and $[P]$ in the following way:

$$
\begin{aligned}
{\left[Q_{l}\right] } & =\left[C_{\imath \jmath}\right]\left[V_{J}\right] \\
{\left[V_{l}\right] } & =\left[P_{\imath \jmath}\right]\left[Q_{J}\right] \quad\left[P_{\imath \jmath}\right]^{-1}=\left[C_{\imath j}\right] .
\end{aligned}
$$

The $i, j$ th element of the $[C]$ matrix is the free charge per unit length on the $i$ th conductor when all conductors except the $j$ th one are grounded and the $j$ th conductor is charged to a potential of $1 \mathrm{~V}$. Hence, the elements of $[C]$ can be determined by relating the charge on the conductors to their potentials. However, in our work, we will take a different approach, one based on energy calculations. In this way, we can take advantage of the variational nature of the energy expressions. For an arbitrary distribution of charges on the strips, the electrostatic energy stored in the system per unit length can be expressed as

$$
U=\frac{1}{2}\left[Q_{l}\right]\left[P_{l J}\right]\left[Q_{j}\right]=\frac{1}{2}\left[V_{l}\right]\left[C_{l j}\right]\left[V_{J}\right]
$$

Note that $[P]$ can be expressed in terms of the electrostatic energy for different situations in the following way:

$$
\begin{aligned}
& P_{l l}=2 U^{i l} / Q_{\imath}^{2} \\
& P_{\imath \jmath}=\left(U^{i j}-U^{u}-U^{j \jmath}\right) / Q_{\imath} Q_{\jmath}, \quad i \neq j
\end{aligned}
$$

where $U^{i j}$ is the electrostatic energy stored in the system per unit length when all conductors except the $i$ th and $j$ th ones are isolated and discharged and the $i$ th and $j$ th conductors support charges $Q_{\imath}$ and $Q_{j}$, respectively. Now, our problem is the evaluation of the energy for the $N+\left(\begin{array}{c}n_{c} \\ 2\end{array}\right)$ different distributions of charges necessary to compute all the elements of the $[P]$ matrix (and consequently the $[C]$ matrix).

The electric energy can be expressed in the Fourier domain as follows:

$$
\begin{aligned}
U= & \frac{1}{2 b} \sum_{n=1}^{\infty}\left[\tilde{\rho}_{s}(n)\right]^{t}\left[\tilde{G}_{s r}(n)\right]\left[\tilde{\rho}_{r}(n)\right] \\
M= & \text { number of interfaces with conducting strips } \\
& (M=1 \text { for coplanar strips }) \\
s, r= & 1, \cdots, M
\end{aligned}
$$

where $\tilde{\rho}_{J}(n)$ is the Fourier transform of the surface charge density on the $j$ th interface (the expressions (1) have been used in (5)). A knowledge of these distributions of charges is only possible in very limited cases. However, the stationary nature of (5) allows us to apply Ritz's minimization procedure to obtain a very accurate estimation of $U$.

Let $n_{s}$ be the number of conducting strips lying on the $s$ th interface. The surface charge density can be expanded in a set of basis functions:

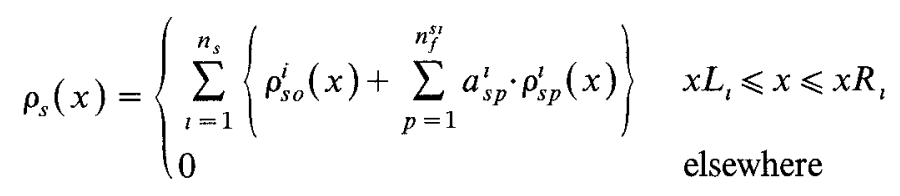

where

$$
\int_{0}^{b} \rho_{s 0}^{l}(x) \cdot d x=1
$$

and

$$
\int_{0}^{b} \rho_{s p}^{t}(x) \cdot d x=0 .
$$

The variational coefficients $a_{s p}^{J}$ are obtained by minimization of the electrostatic energy in (5) (Ritz's procedure). This process leads to the following system of linear equations:

$$
\sum_{s=1}^{M} \sum_{j=1}^{n_{s}} \sum_{p=1}^{n_{j}^{s j}} A_{q p}^{i j}(r, s) \cdot a_{s p}^{J}=B_{q}^{i}(r)
$$

where the coefficient matrix is expressed in terms of the Fourier transforms of the basis functions in the following way:

$$
A_{q p}^{i j}(r, s)=\sum_{n=1}^{\infty} \tilde{\rho}_{r q}^{i}(n) \cdot \tilde{G}_{r s}(n) \cdot \tilde{\rho}_{s p}^{J}(n)
$$

and the independent terms column is related to the particular distribution of charged and isolated strips to be considered for each energy calculation. To determine the inverse of the capacitance matrix from (4), it is clear that we must only consider cases with one or two strips charged 


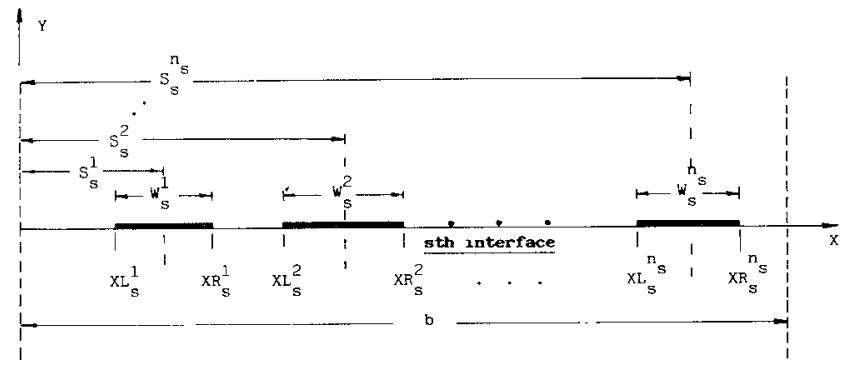

Fig. 2. Detail of the $s$ th interface of the structures in Fig. 1.

and the rest isolated and without charge. Therefore, if the $l$ th strip of the $u$ th interface and the $m$ th strip of the $v$ th interface are charged, the independent terms of the equation system will be given by

$$
B_{q}^{t}(r)=\sum_{n=1}^{\infty} \tilde{\rho}_{r q}^{t}\left\{\tilde{G}_{r u} \tilde{\rho}_{u o}^{1}+\tilde{G}_{r o} \tilde{\rho}_{v o}^{m}\right\} .
$$

Once (7) is solved, the electric energy per unit length can be computed from the following expression:

$$
\begin{aligned}
U^{l m}(u, v) & =\frac{1}{2 b}\left\{\sum _ { n = 1 } ^ { \infty } \left\{\tilde{G}_{u u}\left|\tilde{\rho}_{u o}^{l}\right|^{2}+\tilde{G}_{v v}\left|\tilde{\rho}_{v o}^{m}\right|^{2}\right.\right. \\
& \left.\left.+2 \cdot \tilde{G}_{u v} \tilde{\rho}_{u o}^{l} \tilde{\rho}_{v o}^{m}\right\}-\sum_{s=1}^{M} \sum_{J=1}^{n_{s}} \sum_{p=1}^{n_{j}^{s}} a_{s p}^{J} \cdot B_{p}^{J}(s)\right\} .
\end{aligned}
$$

Expression (10) is evaluated for all possible values of $u, v=1, \cdots, M$ and $l, m=1, \cdots, n_{s}$; the $[P]$ coefficients can then be obtained by using (4). This process must be carried out for the structure with and without dielectric layers to obtain $\left[C_{i j}\right]$ and $\left[C_{i j}^{v}\right]$. In this way, the capacitance and inductance matrices are obtained and the system is completely characterized under a quasi-TEM operation.

\section{Trial Functions}

Before generating numerical results, a good choice of trial functions is necessary. Different sets of basis functions have been considered in this work. From this study, we conclude that an adequate choice of trial functions must take into account the singularities of the charge distributions at the edges of the strips.

A very simple set of functions is to consider a constant term covering the total charge on the strip and two terms for the singularities at the edges (see Fig. 2):

$$
\begin{aligned}
& \rho_{s 0}^{l}(x)=\frac{1}{W_{s}^{l}} \quad \rho_{s 1}^{l}(x)=\left\{\frac{1}{\sqrt{\frac{x-x L^{i}}{W_{s}^{l}}}}-2\right\} \cdot \frac{1}{W_{s}^{l}} \\
& \rho_{s 2}^{l}(x)=\left\{\frac{1}{\sqrt{\frac{x R^{l}-x}{W_{s}^{i}}}}-2\right\} \cdot \frac{1}{W_{s}^{l}} .
\end{aligned}
$$

This choice is good enough to yield very accurate results for coplanar strip structures. This fact has been concluded from systematic comparisons with previously published results and with the data generated by using a more complete set of basis functions such as the following one (Fig. 2):

$$
\begin{aligned}
\rho_{s 0}^{l}(x)= & \frac{2}{\pi W_{s}^{l}}\left\{\frac{1}{1-\left[\frac{\left(x-S_{s}^{l}\right)}{\left(W_{s}^{l} / 2\right)}\right]^{2}}\right\}^{1 / 2} \\
\rho_{s p}^{l}(x)= & \frac{\pi}{2} \rho_{s 0}^{l}(x) \cdot\left\{\cos \left(\frac{p \pi}{W_{s}^{l}} \cdot\left(x-x L_{s}^{l}\right)\right)\right. \\
& \left.-\cos \left(\frac{p \pi}{2}\right) J_{0}\left(\frac{p \pi}{2}\right)\right\} .
\end{aligned}
$$

In a general case involving broadside couplings, the charge distribution is more complicated and one must use the expansion in (12) to obtain good results. The convergence of the Fourier series is slower with these functions, but the accuracy in certain cases is improved substantially. In order to improve the convergence, the asymptotic performance of the series appearing in (8) and (9) has been considered in the computer programs. In this way, the number of Fourier terms necessary to obtain the desired accuracy is drastically reduced and both choices of trial functions are suitable for quick calculations. CPU time is less than one second per conductor strip for multistrip structures on a VAX11/780 computer.

\section{Numerical Results}

The theory presented in this paper was used to write a computer program which provides the capacitance and inductance matrices for a system of coupled strips embedded in a multilayered isotropic or anisotropic medium. From these matrices, mode impedances and phase velocities are readily obtained [3]. In order to check the results, we analyzed several particular structures previously studied by other authors. In the following paragraphs, we show these comparisons and some new results.

In order to show the effect of a good choice of basis functions we compare our results with the ones reported by Koul and Bhat [12]. (These authors provide a useful method to analyze a wide variety of symmetrical striplike structures on anisotropic substrates.) In Fig. 3 we compare the results obtained in [12] for the interelectrode capacitance of a coplanar structure (electro-optic modulator) with the ones calculated by means of the method in this paper. We show two curves by using the trial functions in (12) with $n=0$ and $n=5$. In both cases we found a very good agreement for narrow strips. However, a significant discrepancy is observed for wide ones. In our opinion, this discrepancy is due to the fact that the trial function used in [12] cannot conveniently represent the charge distribution on wide strips. These curves were also computed by using (11) and the discrepancy was less than 0.5 percent for all 


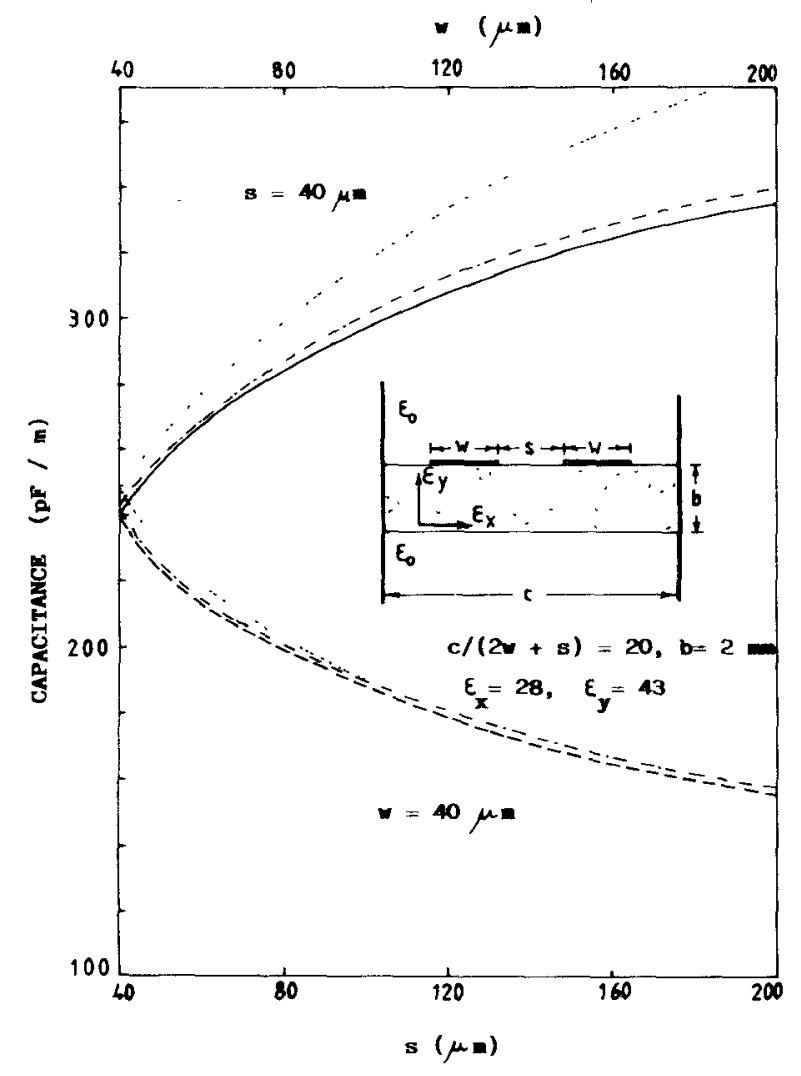

Fig. 3. Interelectrode capacitances of electro-optic modulator as a function of the width of the strips and their separation. --- and are the results reported in [12]. - - - - represent our computations with one basis function, and $\cdots$ represent our computations with five basis functions.

dimensions. This fact confirms that (11) is a good set of basis functions for coplanar strips.

The structures shown in Fig. 4(a) and (b) were analyzed by Kitazawa and Mittra [7] and by Kitazawa and Hayashi [8]. The effective dielectric constants for $C$ and $\pi$ modes of these structures are represented in Figs. 5 and 6. As we can see, the results in [7] and [8] are in good agreement with our data.

Table I compares our data with those obtained by Wei et al. [10] for the case of two coupled microstrip lines between two ground planes in a homogeneous and isotropic medium. Table II shows the results obtained for a structure with three strips embedded in a three-layered dielectric medium between two ground planes. This structure was also studied by Wei et al. in [10]. We compare both results in Table II. Significant differences are detected, but these are within the margin of error given in [10].

More exact calculations were previously reported by Kammler on multiconductor structures in a homogeneous and isotropic medium [9]. In Table III, we show the results obtained in the analysis of a pair of asymmetrical coupled strips between two ground planes. An excellent agreement (within \pm 0.002 in all cases) was found. (It must be emphasized that the Kammler results are exact to within \pm 0.001 .) These data were generated with the trial functions in (12). A typical discrepancy of 1 percent to 5 percent was observed by using (11). However, the set of

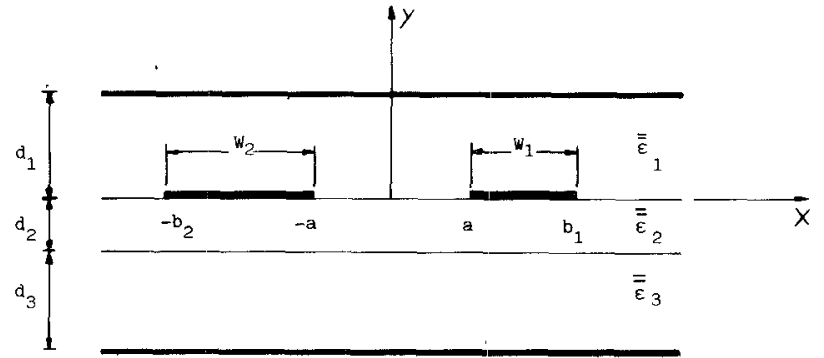

(a)

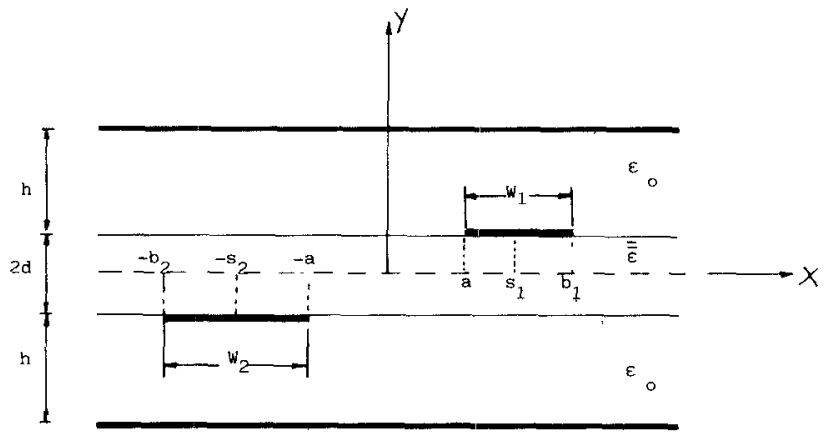

(b)

Fig. 4. (a) Cross-sectional view of asymmetrical coupled strip lines. (b) Cross-sectional view of broadside-coupled strip lines of unequal width.

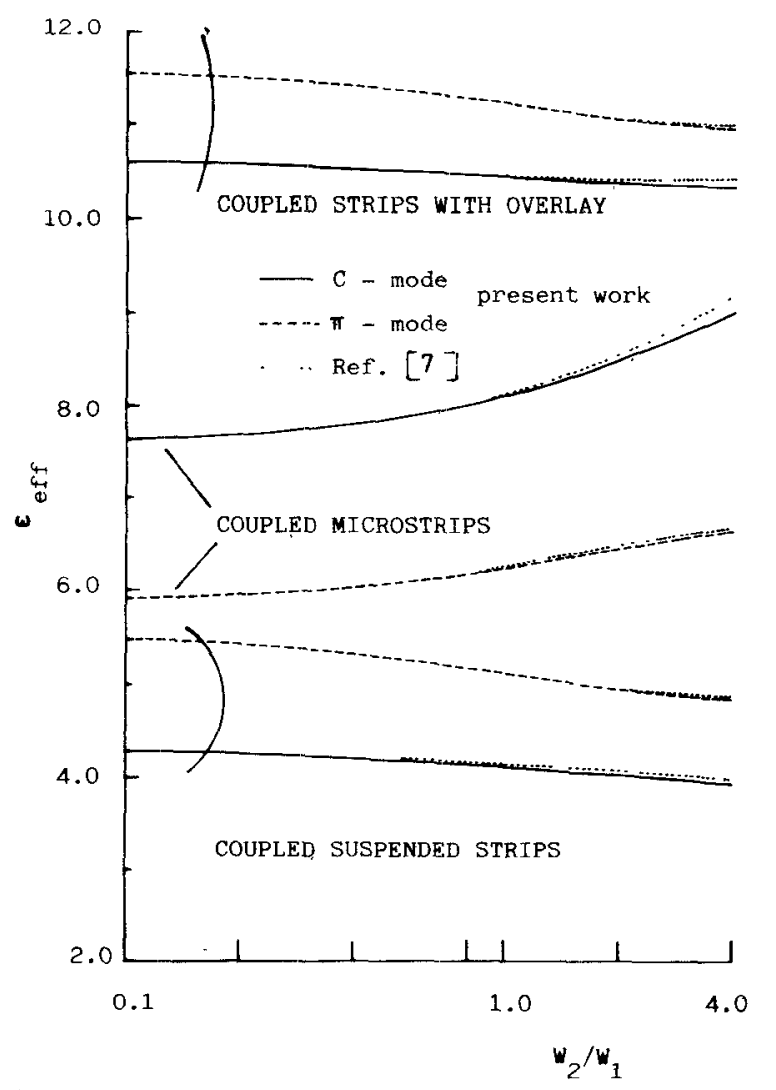

Fig. 5. Quasi-static characteristics versus $W_{2} / W_{1}$. (The dimensions and dielectric constants have been taken from [7]:)

basis functions in (11) allows us to obtain very good results in the case of coplanar structures. In this way, Table IV compares our results for the case of several multiconductor, coplanar structures with those reported in [9]. The 


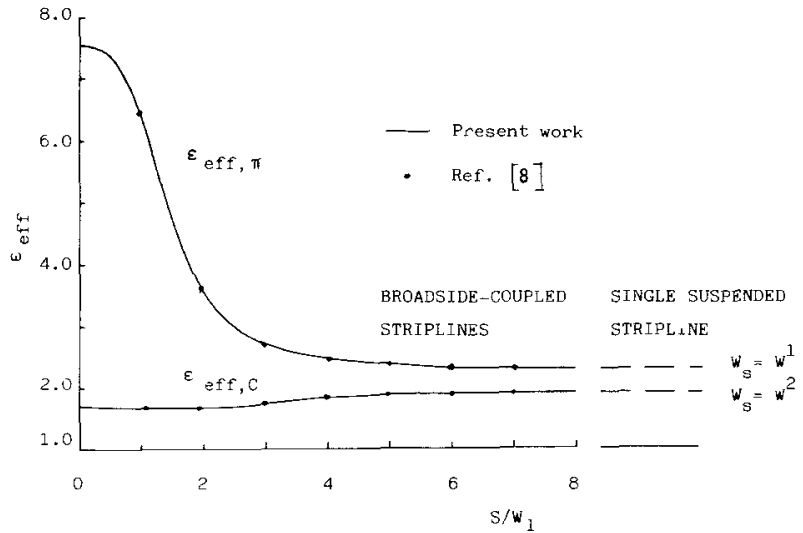

(a)

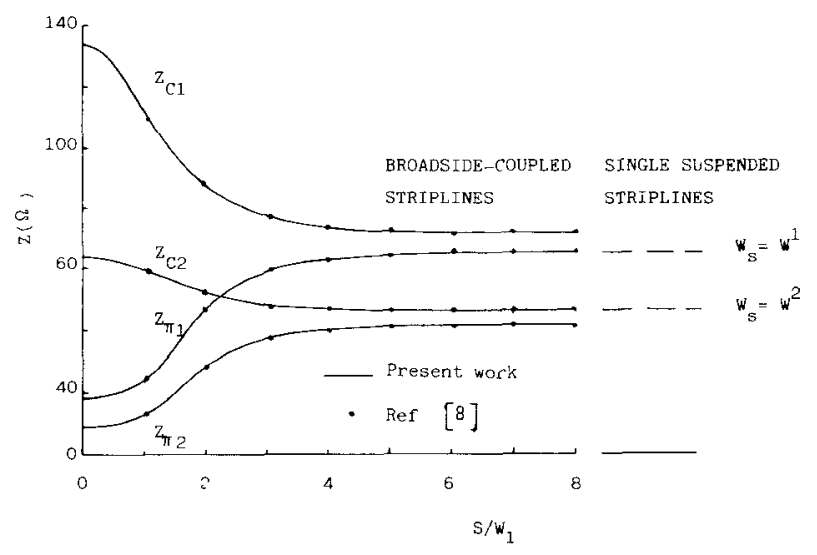

(b)

Fig. 6. (a) Effective dielectric constant versus $S / W_{1}$. (b) Characteristic impedances versus $S / W_{1}$. (Dimensions and dielectric constants have been taken from [8].)

TABLE I

VALUes of THE CAPACITANCE AND INDUCTANCE COEFFICIENTS For TWO COUPLED Microstrip Lines Between Two Ground Planes in a HOMOGENEOUS MEDIUM AND COMPARISON WITH THE DATA REPORTED IN [10].

\begin{tabular}{|c|c|c|}
\hline & & \\
\hline & Ref. $|10|$ & Present work \\
\hline & $0.5356 \times 10^{-9}$ & $0.5320 \times 10^{-9}$ \\
\hline $\mathrm{C}_{11}$ & $0.5 \varepsilon_{0}$ & $-0.1008 \times 10^{-10}$ \\
$\mathrm{C}_{12}$ & $-0.9250 \times 10^{-11}$ & $0.7790 \times 10^{-9}$ \\
$\mathrm{C}_{22}$ & $0.7834 \times 10^{-9}$ & $0.5600 \times 10^{-10}$ \\
$\mathrm{C}_{011}$ & $0.5466 \times 10^{-10}$ & $0.1062 \times 10^{-11}$ \\
$\mathrm{C}_{012}$ & $-0.9439 \times 10^{-12}$ & $-0.5 \times 10^{-10}$ \\
$\mathrm{C}_{022}$ & $0.7994 \times 10^{-10}$ & $0.8200 \times 10^{-6}$ \\
$\mathrm{~L}_{11}$ & $0.2033 \times 10^{-6}$ & $0.1987 \times 10^{-6}$ \\
$\mathrm{~L}_{12}$ & $0.2401 \times 10^{-8}$ & $0.2570 \times 10^{-8}$ \\
$\mathrm{~L}_{22}$ & $0.1390 \times 10^{-6}$ & $0.1357 \times 10^{-6}$ \\
\hline
\end{tabular}

TABLE II

Values of THe CaPacitance and Inductance CofFFicients FOr THREe COUPLED STRIP LiNeS IN A THREe-LAYERED DieleCtric between Ground Planes and Comparison with the Data REPORTED IN [10]

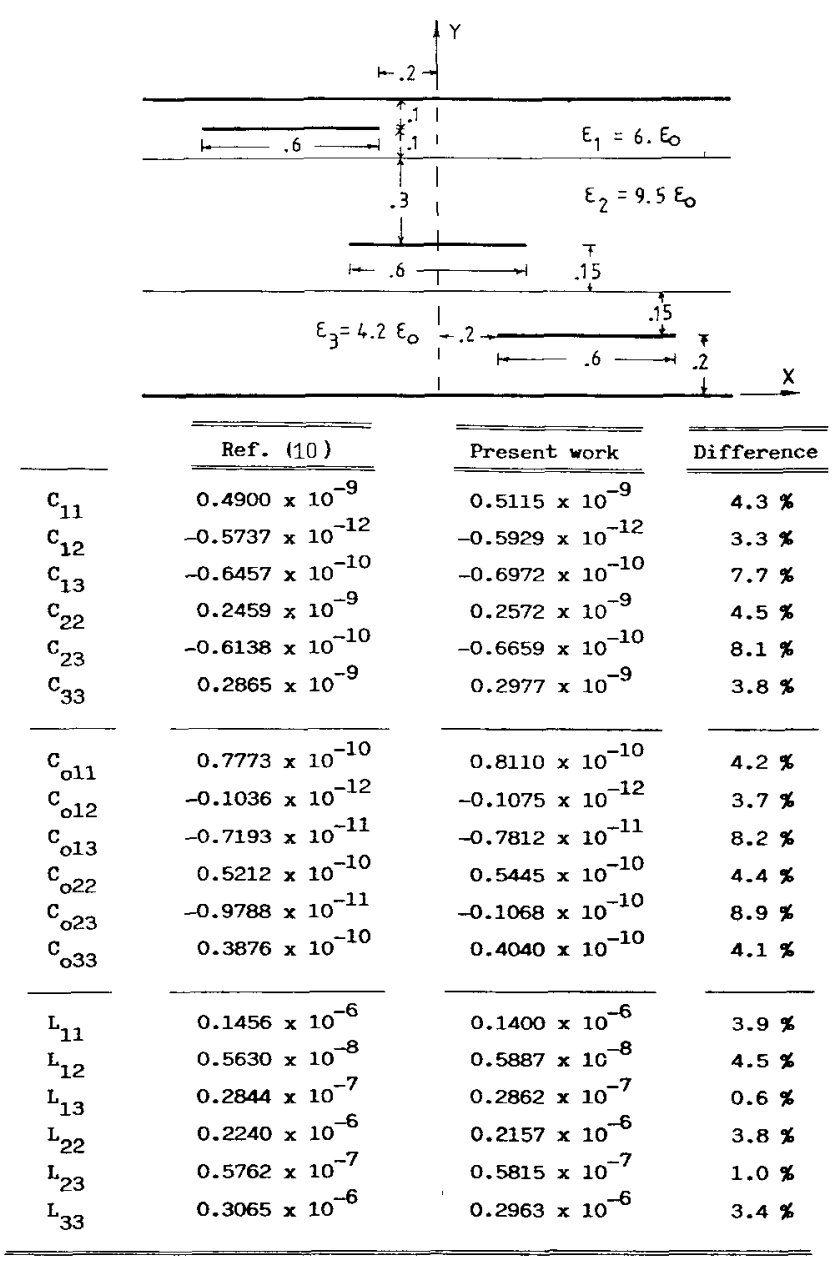

TABLE III

ASYMmetrical BRoadside-Coupled Strips BetWeEN GRound Planes: Comparison with the Capacitance CoffFicients REPORTED BY KAMMLER [9]

\begin{tabular}{|c|c|c|c|c|c|c|c|}
\hline \multirow[b]{2}{*}{$w_{1}$} & \multirow[b]{2}{*}{$w_{2}$} & \multirow[b]{2}{*}{$c_{11} / \varepsilon_{0}$} & \multicolumn{2}{|c|}{$\underline{-w_{1}-1}$} & $\frac{t}{0.2}$ & \multirow[b]{2}{*}{$\mathrm{C}_{12}^{*} \mathrm{\varepsilon}_{\mathrm{o}}$} & \multirow[b]{2}{*}{$\mathrm{c}_{22^{*}}^{*} \varepsilon_{0}$} \\
\hline & & & $c_{12^{\prime}} \tau_{0}$ & $c_{22} \varepsilon_{0}$ & $c_{11}^{*} \varepsilon_{0}$ & & \\
\hline 1.0 & 1.0 & 9.136 & -5.355 & 9.136 & 9.133 & -5.352 & 9.133 \\
\hline 1.0 & 0.8 & 8.862 & -4.743 & 7.760 & 8.860 & -4.742 & 7.759 \\
\hline 1.0 & 0.5 & 8.434 & -3.953 & 6.301 & 8.434 & -3.953 & 6.301 \\
\hline 1.0 & 0.5 & 8.179 & -3.518 & 5.558 & 8.179 & -3.518 & 5.557 \\
\hline 1.0 & 0.4 & 7.904 & -3.064 & 4.807 & 7.903 & -3.063 & 4.807 \\
\hline 1.0 & 0.3 & 7.611 & -2.592 & 4.048 & 7.610 & -2.592 & 4.046 \\
\hline 1.0 & 0.2 & 7.300 & -2.100 & 3.268 & 7.299 & -2.099 & 3.264 \\
\hline 1.0 & 0.1 & 6.956 & -1.561 & 2.424 & 6.956 & -1.561 & 2.423 \\
\hline 1.0 & 0.05 & 6.746 & -1.234 & 1.916 & 6.746 & -1.235 & 1.915 \\
\hline
\end{tabular}


TABLE IV

Capacitance Coefficients Computed for Several Coplanar CONFIGURATIONS AND COMPARISON WITH RESULTS IN KAMMLER [9]

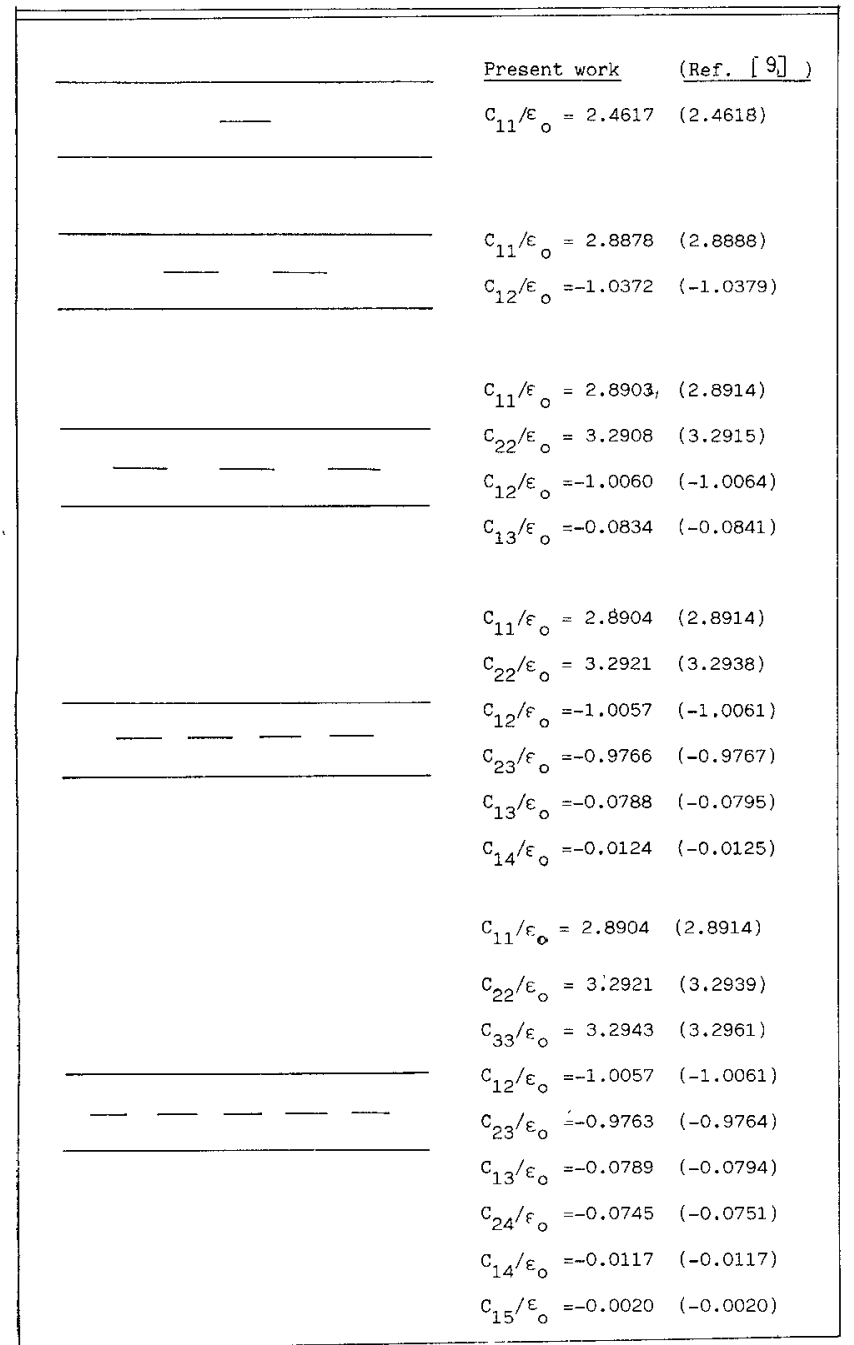

difference is less than 0.05 percent for the worst of the cases studied.

Finally, another configuration was analyzed with our method, one consisting of a three-line symmetrical coupler with a two-layered anisotropic substrate: sapphire $\left(h_{2}\right)$ and pyrolitic boron nitride (P.B.N.) $\left(h_{1}\right)$. Special coupler structures such as this one are often required in communication systems and other microwave applications. In these structures, the quasi-TEM modes (A, B, C) can be propagated [4]-[6]. Fig. 7 represents the dependence of the mode characteristics on the ratio $h_{1} / h$. It can be noticed that there are two values of $h_{1} / h$ that equalize the mode phase velocities. This interesting result is a consequence of the combined effect of the geometry of the structure and the use of anisotropic substrates [17].

\section{CONCLUSIONS}

In this paper the authors have discussed the analysis of shielded multiconductor strip lines embedded in a multilayered anisotropic medium by employing a variational technique in Fourier's discrete domain. The calculation of

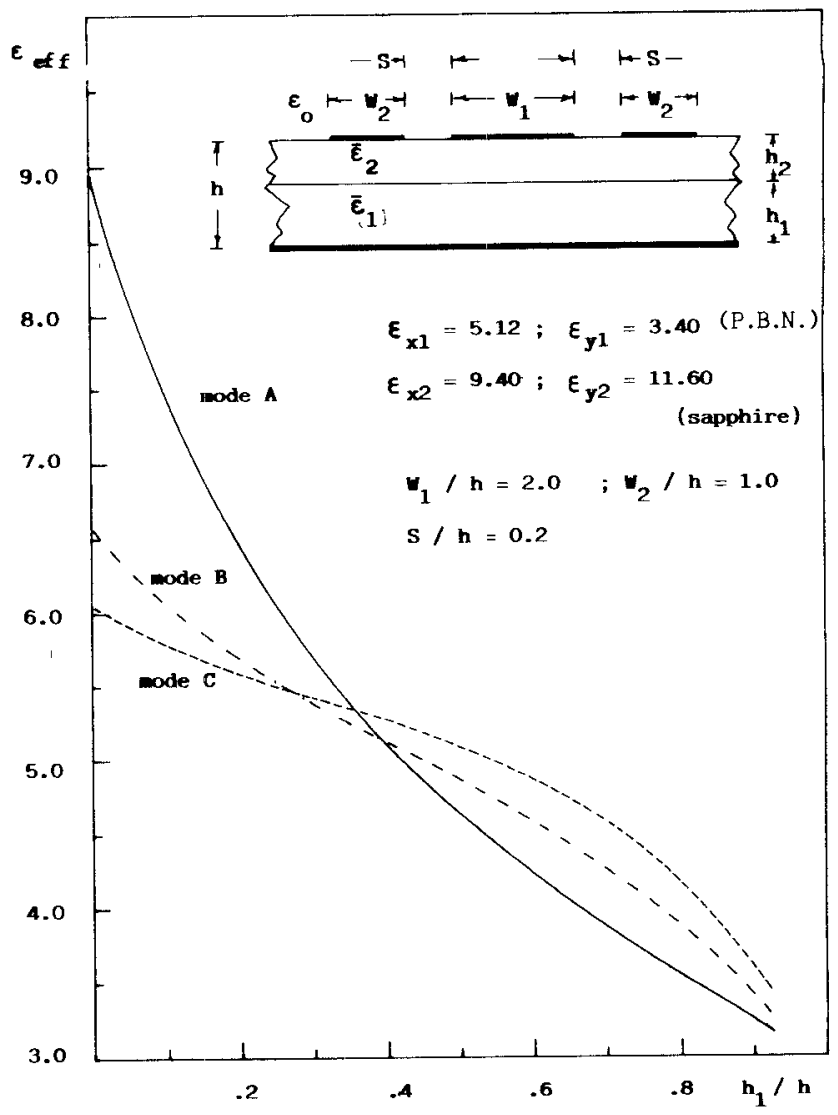

Fig. 7. $\mathrm{A}, \mathrm{B}$, and $\mathrm{C}$ mode effective dielectric constants for three strips on two anisotropic dielectric layers as a function of the relative thickness of each layer.

the capacitance and inductance matrices for lossless configurations is achieved by computing the electric energy per unit length of the structures. The Rayleigh-Ritz procedure has been applied to optimize the solution using adequate trial functions. The method is numerically very efficient and can be easily implèmented in a computer program.

The number of anisotropic dielectric layers and conductor strips is no longer a difficulty because the Green's function matrix is evaluated by means of a very simple recurrence algorithm. Some examples have' been included to illustrate the strength of the method and its accuracy. The propagation modes of three lines with two anisotropic layers have been studied as a particular application.

\section{REFERENCES}

[1] K. D. Marx, "Propagation modes, equivalent circuits, and char acteristic terminations for multiconductor transmission lines with inhomogeneous dielectrics," IEEE Trans. Microwave Theory Tech. vol. MTT-21, pp. 450-457, July 1973.

[2] I. V. Lindell, "On the quasi-TEM modes in inhomogeneous multiconductor transmission lines," IEEE Trans. Microwave Theory Tech. vol. MTT-29, pp. 812-817, Aug. 1981.

[3] R. Marquès and M. Horno, "Propagation of quasi-static modes in anisotropic transmission lines: Application to MIC lines," IEEE Trans Microwave Theory Tech., vol. MTT-33, pp. 927-932, Oct. 1985.

[4] D. Pavlidis and H. L. Hartnagel, "The design and performance of three-line microstrip couplers," IEEE Trans. Microwave Theory Tech., vol. MTT-24, pp. 631-640, Oct. 1976. 
[5] V. K. Tripathi, "On the analysis of symmetrical three-line microstrip circuits," IEEE Trans. Microwave Theory Tech., vol. MTT-25, pp. 726-729, Sept. 1977.

[6] V. K. Tripathi, "The scattering parameters and directional coupler analysis of characteristically terminated three-line structures in an inhomogeneous medium," IEEE Trans. Microwave Theory Tech., vol. MTT-29, pp. 22-26, Jan. 1981.

[7] T. Kitazawa and R. Mittra, "Analysis of asymmetric coupled striplines," IEEE Trans. Microwave Theory Tech., vol. MTT-33, pp. 643-646, July 1985

[8] T. Kitazawa and Y. Hayashi, "Analysis of unsymmetrical broadside-coupled striplines with anisotropic substrates," IEEE Trans. Microwave Theory Tech., vol. MTT-34, pp. 188-191, Jan. 1986.

[9] D. W. Kammler, "Calculation of characteristic admittances and coupling coefficients for strip transmission lines," IEEE Trans. Microwave Theory Tech., vol. MTT-16, pp. 925-937, Nov. 1968.

[10] C. Wei, R. F. Harrington, J. R. Mautz, and T. K. Sarkar, "Multiconductor transmission lines in multilayered dielectric media," IEEE Trans. Microwave Theory Tech., vol. MTT-32, pp. 439-450, Apr. 1984.

[11] Yu Chang and Chang-Yu Wu, "Extension of Chang-Chang's method to analysis of generalised multilayer and multiconductor transmission-line system," Electron. Lett, vol. 7, no. 2, pp. 45-47, Jan, 1971

[12] S. K. Koul and B. Bhat, "Generalized analysis of microstrip-like transmission lines and coplanar strips with anisotropic substrates for MIC, electrooptic modulator and SAW application," IEEE Trans. Microwave Theory Tech., vol. MTT-31, pp. 1051-1059, Dec. 1983.

[13] F. Medina and M. Horno, "Upper and lower bounds on mode capacitances for a large class of anisotropic multilayered microstrip-like transmission lines," Proc. Inst. Elec. Eng., pt. H, vol. 132, no. 3, pp. 157-163, June 1985

[14] F. Medina and M. Horno, "Determination of Green's function matrix for multiconductor and anisotropic multidielectric planar transmission lines," IEEE Trans. Microwave Theory Tech., vol. MTT-33, pp. 933-940, Oct. 1985.

[15] A. Sawicki and K. Sachse, "Lower and upper bound calculations on the capacitance of multiconductor printed transmission line using the spectral domain approach and variational method," IEEE Trans. Microwave Theory Tech., vol. MTT-34, pp. 236-244, Feb. 1986.

[16] N. G. Alexopoulos, "Integrated-circuit structures on anisotropic substrates," IEEE Trans. Microwave Theory Tech., vol. MTT-33, pp. $847-881$, Oct. 1985.
[17] N. G. Alexopoulos, S. Kerner, and C. M. Krowne, "Dispersionless coupled microstrip over fused silica-like anisotropic substrates," Electron. Letter, vol. 12 , no. 22, pp. 579-580, Oct. 28,1976

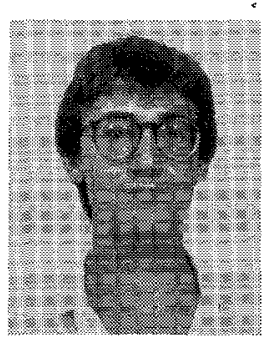

Francisco Medina was born in Puerto Real, Spain, on November 9, 1960. He received the Licenciatura degree in physics from the University of Seville, Spain, in 1983.

$\mathrm{He}$ is currently Assistant Professor of Electricity and Magnetism in the Department of Electricity and Electronics, University of Seville, where he is studying for the Ph.D. degree. His current interest is in multiconductor planar transmission lines and MIC design.

$\mathbf{2}$

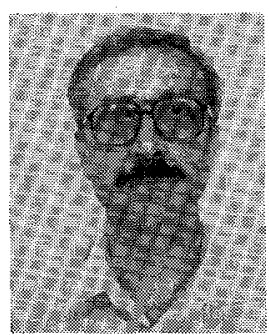

Manuel Horno (M'75) was born in Torre del Campo (Jaén), Spain, on June 29, 1947. He received the degree of Licenciado in physics in 1969 and the degree of Doctor en Ciencias in Physics in 1972, both from the University of Sevilla, Spain.

Since October 1969 he has been with the Department of Electricity and Electronics at the University of Sevilla, where he became an Assistant Professor in 1970, Associate Professor in 1975, and Professor in 1986. His main fields of interest include boundary value problems in electromagnetic theory, wave propagation through anisotropic media, and microwave integrated circuits. He is presently engaged in the analysis of planar transmission lines embedded in anisotropic materials, multiconductor transmission lines, and planar slow-wave structures. 